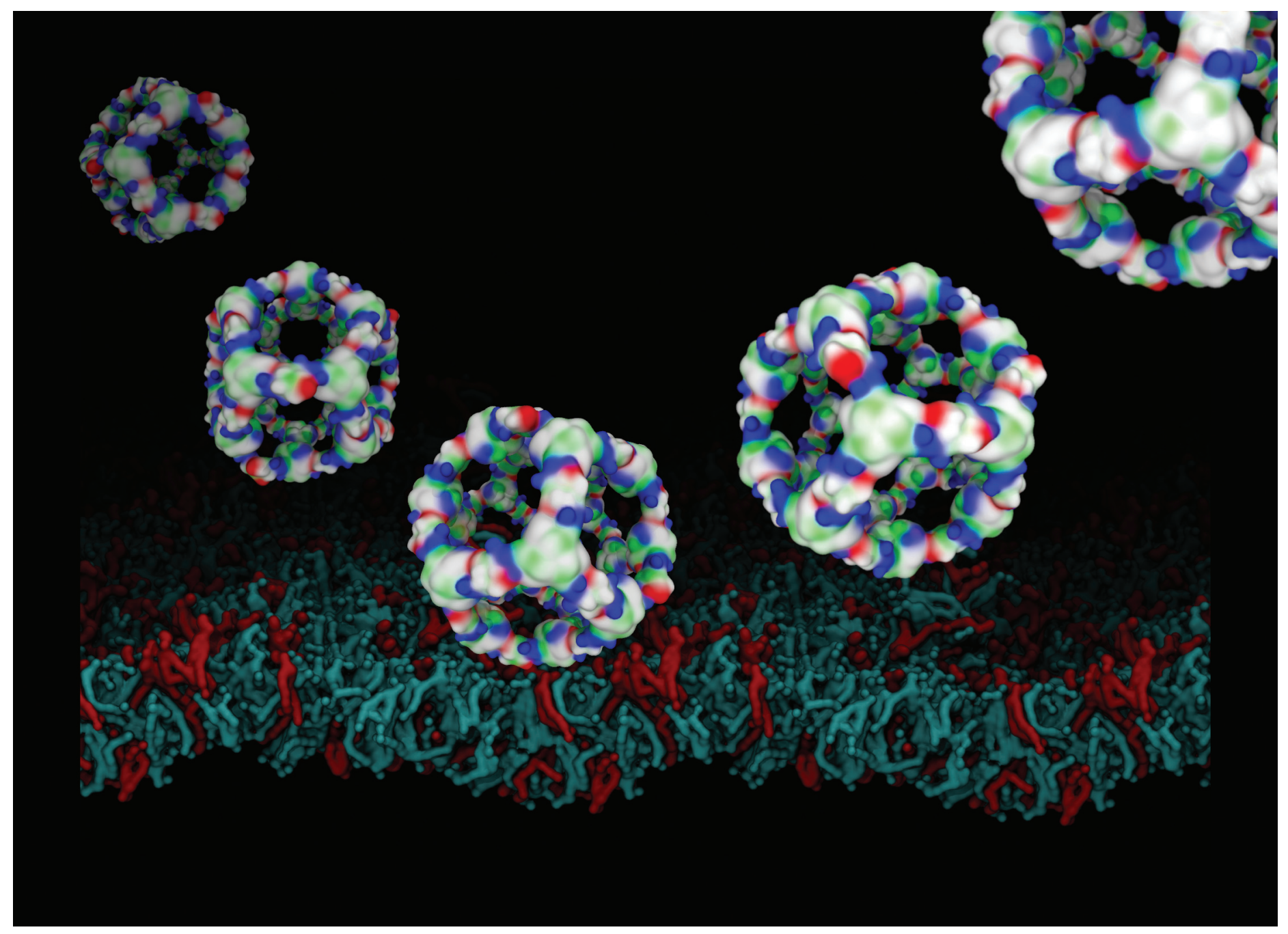

Showcasing research from the Fraternali Franca Laboratory (https://fraternalilab.github.io/) by Irene Marzuoli,

Carlos Cruz, Christian D. Lorenz \& Franca Fraternali.

Nanocapsule designs for antimicrobial resistance

Nanocapsules were placed in an aqueous environment near a membrane (mammalian or microbial) to study the molecular mechanisms leading to antimicrobial effects. The assembly and stability of the nanocapsules were tested via multiscale molecular simulations in water and in bacterial membrane-simulating lipids. Different parametrizations for both capsule and lipids were compared and discussed.

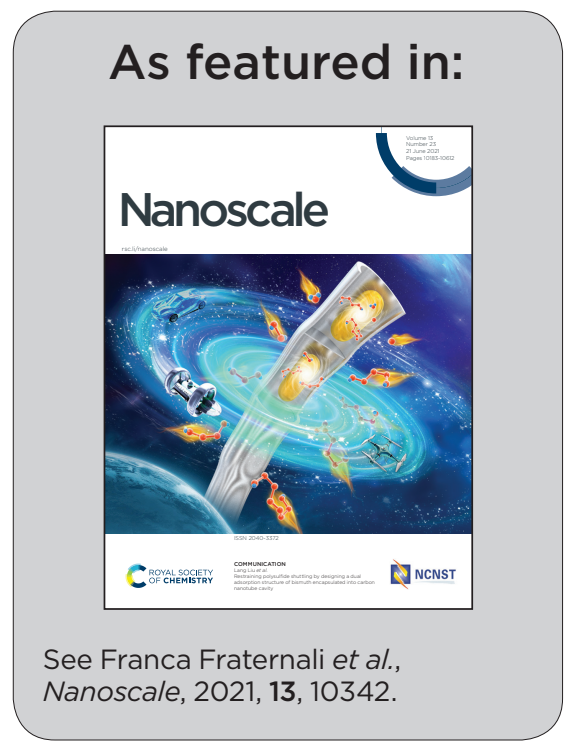


Check for updates

Cite this: Nanoscale, 2021, 13, 10342

\title{
Nanocapsule designs for antimicrobial resistance $\uparrow$
}

\author{
Irene Marzuoli, ${ }^{\mathrm{a}}$ Carlos H. B. Cruz, $\neq^{\mathrm{a}}$ Christian D. Lorenz (D) ${ }^{\mathrm{b}}$ and \\ Franca Fraternali (D) *a
}

Received 15th November 2020,

Accepted 3rd April 2021

DOI: 10.1039/d0nr08146a

rsc.li/nanoscale

\begin{abstract}
The pressing need of new antimicrobial products is growing stronger, particularly because of widespread antimicrobial resistance, endangering our ability to treat common infections. The recent coronavirus pandemic has dramatically highlighted the necessity of effective antibacterial and antiviral protection. This work explores at the molecular level the mechanism of action of antibacterial nanocapsules assembled in virus-like particles, their stability and their interaction with mammal and antimicrobial model membranes. We use Molecular Dynamics with force-fields of different granularity and protein design strategies to study the stability, self-assembly and membrane poration properties of these nanocapsules.
\end{abstract}

\section{Introduction}

In the past decades the constant threat of antimicrobial resistance encouraged the development of novel antimicrobial strategies. ${ }^{1}$ Antimicrobial peptides (AMPs) are highly versatile biomolecules able to disrupt bacterial activity in a plethora of different ways, ${ }^{2,3}$ mostly leading to perturbation and/or poration of the bacterial membrane. ${ }^{2,4,5}$ These biologics qualify as a novel weapon against drug-resistant bacteria because their spectrum of action is substantially different from that of small molecule drugs: this not only opens new therapeutic possibilities, but the characteristics of their broad range action suggest a slower development of resistance once they are employed against common pathogens. ${ }^{6,7}$

Several effective synthetic AMPs have been developed in the past, exploiting information from the naturally occurring ones. In a straightforward approach, natural sequences can be used as templates to generate more powerful or selective peptides via iterative single-point mutations. ${ }^{8-11}$ More generally, virtual screenings of thousands of AMPs have highlighted recurring patterns connected with their activity, ${ }^{12-15}$ and the physicochemical properties inducing their function started to be eluci-

\footnotetext{
${ }^{a}$ Randall Centre for Cell and Molecular Biology, King's College London, London, UK. E-mail: franca.fraternali@kcl.ac.uk

${ }^{b}$ Department of Physics, King's College London, London, WC2R 2LS, UK

$\dagger$ Electronic supplementary information (ESI) available: Additional information on the capsules; alignment of Wild Type and modified $\beta$-annulus; radius of gyration of the capsules simulated; area per lipid, component density and lipidtail order of membrane simulations (no capsules); side chains/backbone hydrogen bonds for atomistic simulation of capzip capsule; radial distribution function of capzip and $\beta$-annulus capsules amino acids around bacterial membrane lipid moieties. See DOI: 10.1039/D0NR08146A

¥Present address: Instituto de Tecnologia Química e Biológica António Xavier, Universidade Nova de Lisboa, Oeiras, Portugal.
}

dated by biophysical studies in vitro but also in silico, e.g. employing Molecular Dynamics simulations. ${ }^{10,16,17}$

Combining this available knowledge with protein engineering techniques, it is possible to design AMPs that perform additionally desired functions, such as self-assembly into organised objects: ${ }^{18-21}$ this can boost the antimicrobial activity of the peptide by enhancing its local concentration, generating premises for effective drug or gene delivery. The success of such a design strategy depends on an accurate knowledge of the molecular-level interactions the peptide has with its copies and with the bacterial membrane. Even for systems with proven antimicrobial activity, a deep understanding of these details is beneficial to set in place design strategies for activity enhancement.

Molecular Dynamics (MD) simulations have been extensively used in the past to elucidate the mechanisms of action of AMPs, where experiments were insufficient to fully uncover their details. ${ }^{22-32}$ Ongoing advances in computing power are continuously increasing the potential of MD simulations, ${ }^{33,34}$ which have contributed to clarify how peptides translocate through membranes, ${ }^{25,26}$ how they oligomerise before translocation, ${ }^{27}$ and to identify the role of specific amino acids and secondary structures in interacting with bacterial membranes. ${ }^{28,29}$

The development of models for bacterial membranes plays a crucial role in the success of such investigations. The simplest ones are bilayers composed of a few phospholipids species with an overall negative charge, ${ }^{31,35,36}$ to capture both the hydrophobic environment and the characteristic electrostatic interaction that AMPs have to overcome to penetrate into the bacterial cytoplasm. These bilayers can also be experimentally assembled and their interactions with AMPs measured with precision, ${ }^{18,37,38}$ which makes the comparison with MD simulations possible, as exploited in this work. 
A more faithful representation of bacterial membranes requires the parametrisation of a greater variety of such phospholipids (e.g. phosphocholines, phosphoetilamine, phosphoglycerols) ${ }^{39-43}$ together with peptidoglycans (PG) ${ }^{44,45}$ and lipopolyaccharides (LPS). ${ }^{46-48}$ The combination of these molecules allows to represent the Gram positive bacteria cell membrane (a double layer of phospholipids coated with long PG chains), ${ }^{49}$ and the double membrane of Gram negative ones (an inner membrane of phospholipids, and an outer one formed by phospholipids at the interior and LPSs externally, with the two interspaced by a layer of PGs). ${ }^{49}$ LPSs are therefore of particular interest as the first molecules approached by external agents, such as AMPs, when attacking Gram negative bacteria.

As the translocation of AMPs and other membrane-penetrating nanoparticles requires long simulation time, the introduction of an electric field across the two sides of the membrane is a useful procedure not only to model the transmembrane potential, but also to accelerate penetration. ${ }^{41,50-54}$ This external perturbation causes the membrane itself to be more prone to disruption, ${ }^{51}$ and pushes positively charged nanoparticles/peptides toward the membrane. ${ }^{52-54}$ The effect of an electric field can also be assessed experimentally and compared with the simulations data, ${ }^{55}$ but the intensity of the field should be kept within physiological values.

Several penetration mechanisms are known, in presence or absence of electric field, and their understanding is crucial for the design of new AMPs. For example, short helical AMPs insert vertically in the membrane and gather together to form a water channel; ${ }^{2,56-58}$ nanoparticles strongly attach to the membrane enhancing its curvature until disruption; ${ }^{48,59}$ additionally Arginine rich peptides exploit the local defects of the membrane to pass through it despite their high charge, as proven by MD simulations. ${ }^{28}$ Finally, transmembrane proteins on the outer Gram negative membrane can facilitate the penetration of some AMPs. Nevertheless models of these systems are computationally expensive and require large scale simulations. ${ }^{60-62}$

A peptide with established assembly and antimicrobial activity is capzip, ${ }^{18,63}$ which self-assembles into hollow capsules with a predominant size range of $20-200 \mathrm{~nm}$, and derives its antimicrobial activity from a short six amino acid sequence of the antimicrobial portion ${ }^{64-66}$ of bovine Lactoferrin (PDB code: 1LFC), mutated to favour self-assembly (Fig. 1). Molecular Dynamics simulations have helped identifying the assembled structure and how it approaches the membrane ${ }^{63}$ but further investigation is necessary to identify the determinants of its selectivity between mammalian and bacterial membranes, and to quantify the perturbations imposed on the latter. A characteristic of the capzip peptide is its branched sequence, constituted by a central scaffold and three short arms without any secondary structure. The arms both favour the pairing with other copies of the molecule by antiparallel $\beta$-sheet formation, and bear the positive charge known to perturb the bacterial membrane. ${ }^{2,5,67}$

The design of self-assembling peptides is an established field which has successfully led to the design of multi-purpose

\section{CAPZIP}
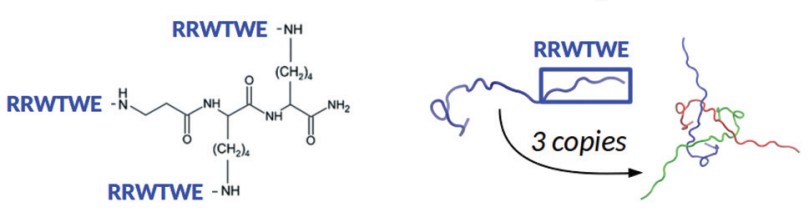

AM- $\beta$-ANNULUS

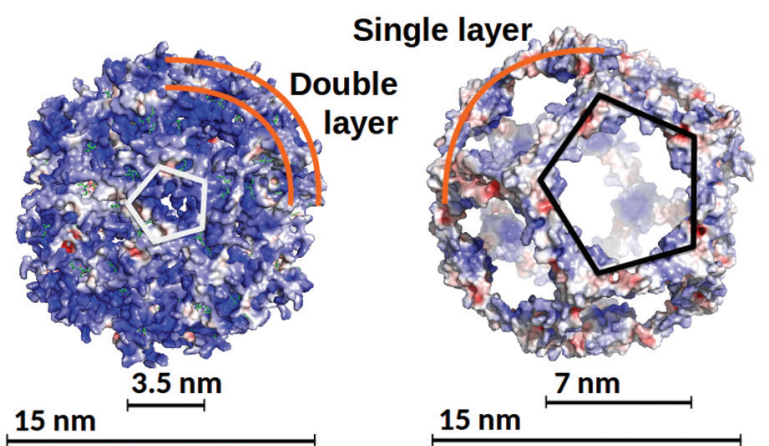

Fig. 1 (Top) Capzip peptide (which constitutes the capsule building block), and AM- $\beta$-annulus peptide, with the respective capsule building block, constituted by three AM- $\beta$-annulus peptides. (Bottom) Modelled antimicrobial capsules, capzip on the left and $A M-\beta$-annulus on the right. Capsules are shown in surface representation, colour coded by surface charge with blue positive, white neutral, and red negative charge (pyMol software ${ }^{89}$ ). The diameter of the modelled antimicrobial capsules and of the holes on their surfaces are indicated.

materials. $^{68-72}$ The interesting results we obtained for the capzip system by the use of combined simulations and experimental investigations encouraged the in silico design of a new antimicrobial capsule obtained by grafting the same six amino acid antimicrobial motif composed from the capzip peptide onto a new scaffold, the $\beta$-annulus construct from the $\mathrm{C}$ chain of the Tomato Bush Stunt virus protomer (TBSV, PDB code $\left.2 \mathrm{TBV}^{73}\right)$. This is a peptide of 24 amino acids sequence which, in the original viral structure (hosting several other proteins), assembles with two other copies of itself to provide a triangular block with three extended arms, similar to the capzip peptide.

The 24-mer alone was shown in previous work to selfassemble into hollow capsules both in its native state ${ }^{74}$ and when functionalised with additional chemical entities, such as gold or zinc nanoparticles, ${ }^{75,76}$ short DNA strands ${ }^{77}$ or peptides. ${ }^{78,79}$ In our design, the RRWTWE antimicrobial sequence was transplanted onto the extended arms of the $\beta$-annulus to ensure antimicrobial activity. It should be noted that the resulting AM- $\beta$-annulus capsule has a different ratio between the antimicrobial portion and the non functional moiety joining the arms with respect to the capzip capsule.

In this context, the present work establishes a pipeline of molecular modelling and multi-scale Molecular Dynamics simulations (from atomistic to coarse-grained) to study the stability of the capzip and AM- $\beta$-annulus capsules in water, and their interaction with model membranes. Our computational approach can be used as a framework to test novel antimicrobial self-assembling compounds and their mode of action. 


\section{Methods}

\section{Capzip modelling and simulations}

Following up from previous work, ${ }^{63}$ we performed atomistic and coarse-grained simulations of a capzip capsule in solution and on model membranes, using the atomistic GROMOS 54a8 force field, ${ }^{80,81}$ and the coarse-grained description provided by MARTINI $^{82,83}$ and SIRAH. ${ }^{84,85}$ All simulations were performed with the GROMACS software, version 5.5 and $2016 .^{86-88}$ Table 1 reports all the simulations performed.

Atomistic simulations. The capzip capsule was modelled in the shape of a double-layered truncated icosahedron, using GROMACS ${ }^{88}$ and MOE $^{90}$ tools as previously reported. ${ }^{63}$ Its geometrical and chemical characteristics are shown in Fig. 1 and ESI Table 1. $\uparrow$ A DLPC/DLPG 3:1 bilayer was selected to model bacterial membranes, consistent with previous in vitro experiments performed on Supported Lipid Bilayers with the capzip peptide: ${ }^{18}$ the negative charge of DLPG captures one of the main characteristics that distinguishes bacterial from mammal membranes, ${ }^{49,91-93}$ and mimics the inner Gram negative membrane. The atomistic coordinates for the membrane were built with the PACKMOL software. ${ }^{94}$ Two patches were built, hosting 512 and 740 lipids respectively. The expected area per lipid was reached during a 400 ns equilibration (see simulations details below), the final configuration

Table 1 Table with an overview of the simulations performed

\begin{tabular}{|c|c|c|}
\hline System $^{a}$ & Force field & Time (ns) \\
\hline Capzip bilayer capsule & GROMOS & 100 \\
\hline Capzip bilayer capsule & MARTINI & 1000 \\
\hline Capzip bilayer capsule & SIRAH & 1000 \\
\hline Capzip monolayer capsule & MARTINI & 1000 \\
\hline Capzip monolayer capsule & SIRAH & 1000 \\
\hline AM- $\beta$-annulus capsule & SIRAH & 1000 \\
\hline \multicolumn{3}{|l|}{ Capzip pentagonal unit on: } \\
\hline Small bact. $\mathrm{m}$. & GROMOS & 500 \\
\hline Large bact. $\mathrm{m}$. & GROMOS & 500 \\
\hline Small bact. m., increasing $\mathrm{E}$ & GROMOS & (Total) 2000 \\
\hline Small bact. m., $130 \mathrm{mV} \mathrm{nm}^{-1}$ & GROMOS & 500 \\
\hline Large bact. m., $130 \mathrm{mV} \mathrm{nm^{-1 }}$ & GROMOS & 500 \\
\hline Bact. m., 130 or $140 \mathrm{mV} \mathrm{nm}{ }^{-1}$ & GROMOS & 500 \\
\hline \multicolumn{3}{|l|}{ Capzip bilayer capsule on: } \\
\hline Bact. m. & MARTINI & 10000 \\
\hline Bact. m., $20 \mathrm{mV} \mathrm{nm}{ }^{-1}$ & MARTINI (P) & 500 \\
\hline Bact. m., $40 \mathrm{mV} \mathrm{nm}{ }^{-1}$ & MARTINI (P) & 500 \\
\hline LPS m. ${ }^{a}$ & MARTINI (P) & 1000 \\
\hline Mamm. m. & MARTINI & 10000 \\
\hline Mamm. m., $20 \mathrm{mV} \mathrm{nm^{-1 }}$ & MARTINI (P) & 500 \\
\hline Mamm. m., $40 \mathrm{mV} \mathrm{nm}{ }^{-1}$ & MARTINI (P) & 500 \\
\hline Bact. m., ${ }^{a} 4 \mathrm{mV} \mathrm{nm}{ }^{-1}$ & SIRAH & 10000 \\
\hline Bact. m., 20 or $40 \mathrm{mV} \mathrm{nm}{ }^{-1}$ & SIRAH (P) & 500 \\
\hline Mamm. m., 20 or $40 \mathrm{mV} \mathrm{nm}{ }^{-1}$ & SIRAH (P) & 500 \\
\hline \multicolumn{3}{|c|}{ AM- $\beta$-annulus monolayer capsule on: } \\
\hline Bact. m., ${ }^{\mathrm{b}} 4 \mathrm{mV} \mathrm{nm}{ }^{-1}$ & SIRAH & 10000 \\
\hline
\end{tabular}

In the System field the following abbreviations are used: bact. $\mathrm{m}$. for bacterial membrane DLPC/DLPG $3: 1$, (except for ${ }^{\mathrm{b}}$ where it is POPC/ POPS $3: 1$ ); LPS $\mathrm{m}$. for bacterial outer membrane LPS (top leaflet) and POPE/POPG/CDL $85: 5: 10$ (bottom leaflet); mamm. m. for mammal membrane (DLPC). The notation (P) for the MARTINI force field indicates the Polar water model. of which was used for simulations with the peptide. To simulate the action of the capzip peptide on the membranes, a pentagonal unit of the double-layered truncated icosahedron (10 molecules overall) was taken as representative of the full capsule, to allow a faster sampling, and simulated in proximity of the membrane plane. For the bacterial membrane model, additional simulations were run with an external electric field pointing from the side hosting the capsid peptides to the opposite side, to mimic the transmembrane potential.

For all atomistic simulations, the systems were solvated with single point charge (SPC) water; ${ }^{95} \mathrm{Na}^{+}$and $\mathrm{Cl}^{-}$ions were added to balance the charge and to reach a salt concentration of $150 \mathrm{mM}$, matching the experimental conditions. The capzip peptide parametrisation was built by comparison with tabulated data for peptidic bonds and amino acid moieties. Lipid parameters were taken from ref. 39 for DLPC, while for DLPG they were built from the POPG ones available in ref. 43 . Temperature was maintained by independently coupling protein, membrane, and solvent (plus ions) to three external temperature baths using a velocity rescaling thermostat ${ }^{96}$ with coupling constant $\tau_{\mathrm{T}}$ of 0.1 ps. Pressure was kept at 1 bar by Berendsen $^{97}$ or Parrinello-Rahman barostats ${ }^{98}$ (for equilibration and production, respectively) using an isotropic or semi-isotropic coupling (simulations without and with membrane), with isothermal compressibility of $4.5 \times 10^{-5} \mathrm{bar}^{-1}$ and coupling constant $\tau_{\mathrm{P}}$ of 1 ps. Electrostatic interactions were treated using the smooth Particle Mesh Ewald algorithm ${ }^{99}$ with a Fourier grid spacing of $0.12 \mathrm{~nm}$, and a short-range cutoff of $1.2 \mathrm{~nm}$; van der Waals interactions were calculated with a $1.2 \mathrm{~nm}$ cutoff. All atomistic runs were performed using a 2 fs time step.

Simulations of the capzip capsule in solution were run for $100 \mathrm{~ns}$, in three replicas. In order to stabilise the structure, an equilibration was run where the initial distances between the paired arms of different capzip peptides (i.e. laying on the same edge of the truncated icosahedron) were restrained with a harmonic potential of $1000 \mathrm{~kJ}(\mathrm{~mol} \mathrm{~nm})^{-1}$ force constant, and progressively released over 150 ns. All the assembled membrane bilayers were first equilibrated for 50 ps at $50 \mathrm{~K}$, then the temperature was gradually increased to 300 over $500 \mathrm{ps}$, and finally a 400 ns production run was performed (using the NPT ensemble). A similar equilibration procedure was followed for peptide-membrane systems. Exploratory simulations on the membranes with an external electric field were run for the bacterial model with 512 lipids as follows: starting from $20 \mathrm{mV} \mathrm{nm}^{-1}$, the field was increased by $20 \mathrm{mV}$ $\mathrm{nm}^{-1}$ every $200 \mathrm{~ns}$, up to electroporation value of the membrane in presence of the peptide. Three replicas were run at the electroporation value to confirm the results, and an additional one on the larger membrane hosting 740 lipids. A similar procedure was performed for the membrane alone. ESI Table 2 and ESI Fig. 4 and $5 \dagger$ reports information on the simulations of the membrane without capzip.

MARTINI simulations. The approach of the whole capzip capsule to model membranes was simulated with a coarsegrained MARTINI force field description, ${ }^{82,83}$ with two replicas 
for both a model bacterial and a model mammal composition. The coarse-grained capsule coordinates were obtained from the atomistic structure using martinize.py ${ }^{100}$ with a customised mapping for the central branched residue. Parameters for this residue were obtained using pycgtool.py. ${ }^{101}$ Bacterial and mammalian model membranes hosting 2880 and 2888 lipids respectively were built with insane.py. ${ }^{102}$ The mammalian model was build with DLPC lipids only, as the zwitterionic composition closest to the bacterial one used (DLPC/DLPG $3: 1$ ). The capzip capsule was initially placed at a minimum distance of $1 \mathrm{~nm}$ from the membrane surface. Simulations were performed with the standard MARTINI water model and $\mathrm{Cl}^{-}$counter ions. The same configuration was simulated with an external electric field (20 or $40 \mathrm{mV} \mathrm{nm}^{-1}$, one replica each) with MARTINI polar water ${ }^{103}$ and solution of sodium chloride in concentration $0.15 \mathrm{mM}$. The effect of the field was tested on pure membranes as a control. Furthermore, a model Gram negative outer membrane was built with CHARMM-GUI: ${ }^{104}$ it hosts in the upper layer 567 LPS molecules, and in the bottom layer a mixture of POPE, POPG and cardiolipin in proportion $85: 5: 10$, for a total of 1512 molecules. ${ }^{34}$ Calcium ions were added to balance the LPS charges, the remaining ones were neutralised with sodium ions, finally sodium chloride ions in concentration $0.15 \mathrm{mM}$ were added to the solvent. After the initial equilibration, the membrane was simulated together with the capzip capsule at the initial distance of $1 \mathrm{~nm}$.

To quantify the different binding propensity of capzip capsule for bacterial versus mammal membrane, we computed the Potential of Mean Force (PMF) by applying an external force to impose penetration, pulling the capzip capsule it across the DLPC/DLPG lipid bilayer (chosen versus the LPS membrane for its minor complexity). We then performed umbrella sampling simulations, with a coarse-grained Polar MARTINI description.

For simulations with the standard water model (DLPC/ LDPG and DLPC membranes without electric field), temperature coupling was performed with a velocity rescaling thermostat $^{96}$ with a coupling constant $\tau_{\mathrm{T}}$ of 1 ps. A semi-isotropic pressure coupling was applied at 1 bar pressure, with $4.6 \times$ $10^{-5}$ bar $^{-1}$ isothermal compressibility, using a Berendsen ${ }^{97}$ or Parrinello-Rahman barostat ${ }^{98}$ (equilibration and production phase respectively) with $\tau_{\mathrm{P}}$ of 2 ps or 12 ps. Coulomb interactions were treated with a Reaction Field scheme ${ }^{105}$ and cut off radius of $1.1 \mathrm{~nm}$, van der Waals interactions with a cut off scheme and the same cut off radius. The relative dielectric constant was set to 15 . Simulations performed with the polar water model (DLPG/DLPC and DLPC with electric field; LPS membrane) were run with the parameters above, except the relative dielectric constant set to 2.5 , and the choice of a Particle Mesh Ewald scheme for long-range Coulomb interactions (1.2 nm cut off radius), in agreement with the setup suggested in ref. 103.

Simulations of the capsule in solution were run for $1 \mu \mathrm{s}$, in three replicas with initial constraints over the arms as performed for the atomistic simulation. The membrane models were equilibrated for $1 \mu \mathrm{s}$ with standard water (DLPC/DLPG and DLPC) or following the standard CHARMM-GUI proto$\mathrm{col}^{104}$ (LPS membrane), and the final configuration was used to build peptide-membrane systems, together with the capsule structure (obtained after a $1 \mu$ s equilibration run). The full systems were energy minimised, equilibrated for $500 \mathrm{ps}$ and production was followed for $10 \mu$ s for capzip capsule - DLPC/ DLPG or DLPC systems with standard water, $500 \mathrm{~ns}$ when they are run with polar water (due to the faster binding of the capsule and the speed-up induced by the electric field); and $1 \mu$ s for the capsule - LPSs system. For the simulation with a $40 \mathrm{mV} \mathrm{nm}{ }^{-1}$ electric field, after pore formation, the pressure coupling was switched to isotropic and the simulation prolonged for $100 \mathrm{~ns}$. Control simulations with electric field of the magnitudes used were performed on the membranes alone (ESI Table $2 \dagger$ ).

For umbrella sampling simulations, we first performed a steered simulation pulling the capsule through each membrane. A force of $10000 \mathrm{~kJ}(\mathrm{~mol} \mathrm{~nm})^{2}$ was applied between the center of mass (COM) of the capsule and the COM of the lipids falling in a circle of radius $12 \mathrm{~nm}$ centred on the projection of the capsule COM on $x y$ plane of the membrane. The pulling rate was $10^{-4} \mathrm{~nm} \mathrm{ps}^{-1}$. From these trajectories, 100 frames were extracted, with the $z$ position of the capsule center of mass roughly $0.2 \mathrm{~nm}$ apart. Each frame was simulated for 15 ns restraining the capsule center of mass in its original position with a force constant of $500 \mathrm{~kJ}(\mathrm{~mol} \mathrm{~nm})^{2}$.

All MARTINI simulations were run with a $20 \mathrm{fs}$ time step.

SIRAH simulations. The recent evolution of lipid parametrisation in the SIRAH force field, ${ }^{85}$ united with its characteristic medium grained resolution between the atomistic and the MARTINI coarse-grained one, ${ }^{84}$ make this force field optimal to proceed further with the simulations of antimicrobial capsules on membranes. For this reason, SIRAH was selected for the investigation of the functionalised AM- $\beta$-annulus. To first verify its suitability for the task, the capzip capsule was simulated in solution and in contact with a model bacterial membrane. Due to the recent introduction of lipids parameters in SIRAH, not DLPC nor DLPG were parametrised in this force field at the time of the work presented here. ${ }^{85}$ To pursue the simulation of the capsules on membranes, POPC and POPS (in ratio $3: 1)$ were selected among the SIRAH available lipids, as POPC is zwitterionic and POPS is negatively charged, reflecting the characteristics of DLPC and DLPG, respectively.

The SIRAH parameters and coarse-grained coordinates were assigned to the protein molecules through the cgconv script, distributed with the force field. The mapping of the central branched residue of capzip from atomistic to coarse-grained beads was performed manually, matching when possible similar building blocks of amino acid side chains. The studied capsules were solvated in aqueous solution of sodium chloride in physiologic concentration (0.15 mM), ensuring the systems' neutrality. After energy minimisation, the systems were heated from 0 to $300 \mathrm{~K}$ over $10 \mathrm{~ns}$ at constant volume (10 fs time step), with protein coordinates constrained to their initial position. Like for the other force fields, initial distances between the paired arms of different capzip peptides were restrained 
with a harmonic potential of $1000 \mathrm{~kJ} \mathrm{~mol}^{-1} \mathrm{~nm}^{-2}$, and progressively released over $80 \mathrm{~ns}$ ( $20 \mathrm{fs}$ time step), then the system was simulated for $1 \mu$ (three replicas). The pressure and temperature were kept constant at 1 bar and $300 \mathrm{~K}$, controlled respectively using a V-rescale thermostat and a Berendsen barostat with 2 ns collision-frequency and 8 ps pressure-coupling time. Short-interactions were treated with a $1.2 \mathrm{~nm}$ cut-off radius, whereas the long-range interactions using Particle Mesh Ewald (PME) method.

The POPC/POPS 3:1 model bacterial membrane (3488 lipids) was built using the CHARMM-GUI membrane builder, ${ }^{104}$ and converted to coarse-grained through the cgconv script. Subsequently it was simulated placing the initially equilibrated antimicrobial capsule at $2 \mathrm{~nm}$ distance from the membrane. The system was solvated as above. The membrane was equilibrated over $0.5 \mu \mathrm{s}$ (20 fs time step) at constant temperature and pressure of $300 \mathrm{~K}$ and 1 bar (now maintained with a semi-isotropic Parrinello-Rahman coupling), with the capsule coordinates constrained to their initial positions (1000 kJ mol${ }^{-1} \mathrm{~nm}^{-2}$ constraints force constant). The constraints were then released gradually in four steps, and the simulation was continued for $1 \mu \mathrm{s}$, until the capsule was stably attached to the membrane. Subsequently, a $4 \mathrm{mV} \mathrm{nm}^{-1}$ electric field was applied along the $z$-axis to simulate the transmembrane potential, and the simulation carried on for $1.5 \mu \mathrm{s}$.

\section{$\beta$-Annulus functional design and simulations}

Previous investigations on $\beta$-annulus (sequence ITHVGGVGGSIMAPVAVSRQLVGS) decorated with gold nanoparticles suggested a geometry compatible with a dodecahedral architecture, consistently with the complete TBSV capsid geometry. ${ }^{75}$ In this light we modelled the assembly according to the same geometry.

The triskelion structure formed by three $\beta$-annulus chains (Fig. 1) was extracted from the Tomato Bushy Stunt Virus (TBSV, PDB code: $2 \mathrm{TBV}^{73}$ ). Twenty triskelion copies were then assembled into a dodecahedral capsule with the C-terminal fragments joined through anti-parallel $\beta$-sheets using the Chimera program. ${ }^{106}$ This resulted in a capsule of $15 \mathrm{~nm}$ diameter (Fig. 2a, and ESI Table $1 \uparrow$ ). Next, the antimicrobial sequence RRWTWE was aligned to the $\beta$-annulus sequence and threaded onto the structure via AlignedThread. ${ }^{107}$ It resulted structurally aligned with the RQLVS stretch at the C-terminal (ESI Fig. 1†), thus forming antiparallel $\beta$-sheets with another triskelion copy (Fig. 2b).

It has to be noticed that the $\beta$-annulus triskelion within the virus structure is co-assembling with other viral proteins and therefore once extracted from its original environment hydrophobic residues are exposed on the surface. To improve the stability and solubility of this $\beta$-annulus triskelion, mutagenic assays based on Position-Specific Score Matrix (PSSM) were performed, leaving the antimicrobial sequence as is (Fig. 2b). Twenty Monte Carlo cycles with 3 PSSM-based design rounds with multiples energy minimizations steps each were performed, evaluating the energy within the full capsule assembly, with ROSETTA scripts. ${ }^{107,109}$ Of them, only mutations with
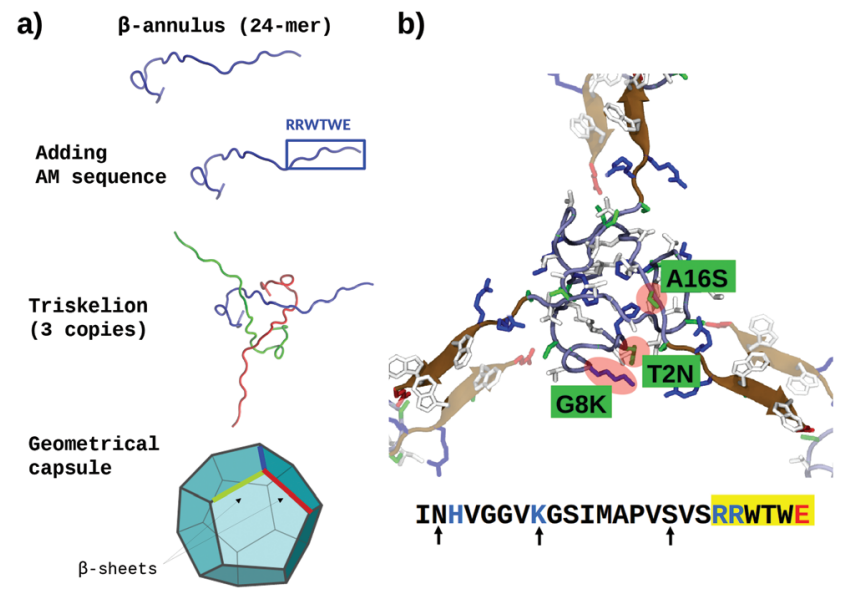

Fig. 2 (a) Schematic of the AM- $\beta$-annulus capsule building strategy. (b) Representation of three $A M-\beta$-annulus sequences forming a vertex of the AM- $\beta$-annulus capsule, with matching arms coming from other vertices (faded). Maroon cartoon shows the RRWTWE antimicrobial sequences. The three residues mutated to improve the solubility are highlighted in red blobs and green panels. Charged amino acid side chains are blue (positive) and red (negative). The sequence of each AM$\beta$-annulus chain is shown at the bottom: antimicrobial sequence highlighted in yellow, mutations indicated by arrows, positive and negative residues in blue and red respectively [VMD software ${ }^{108}$ ].

negative mutational energy (T2N, G8 $\mathrm{K}$ and A16S) were accepted (see Fig. 2b and ESI Fig. $1 \dagger$ ). The characteristics of the resulting capsule are shown in Fig. 1 and ESI Table 1. $\dagger$

The resulting structure (AM- $\beta$-annulus capsule) was simulated with the SIRAH force field in aqueous environment for $1 \mu \mathrm{s}$ and the antimicrobial activity was explored against a POPC/ POPS $3: 1$ membrane (see details in paragraph on SIRAH simulations of capzip capsule). For the latter, the equilibrated AM$\beta$-annulus capsule was initially placed at $2 \mathrm{~nm}$ of distance from the membrane surface. After binding to the membrane (at 500 $\mathrm{ns})$, the simulation was continued for $1 \mu \mathrm{s}$. In this case, only a small electric field ( $4 \mathrm{mV} \mathrm{nm}^{-1}$ ) was necessary to initiate poration. All equilibration procedures are the same as described for the SIRAH simulations of the capzip capsule.

\section{Analysis}

Root Mean Square Deviations (RMSD, with respect to the initial configuration shown in Fig. 1) and radius of gyration $\left(R_{g}\right)$ are computed on the positions of the $\mathrm{C}^{\alpha}$ or coarse-grained backbone beads. The thickness of the capsule wall was computed by fitting a Gaussian on the radial distribution from the centre of the capsule. Hydrogen bonds and Solvent Accessible Surface Area are computed with the native GROMACS tools. The number of conserved arm-arm contacts (which relates loosely to the number of conserved $\beta$-sheets), were computed taking the center of mass (COM) of each antimicrobial arm and counting the contact pairs at less than $1.2 \mathrm{~nm}$ of distance. This holds for both atomistic and coarse-grained representation.

For simulations involving membranes, the area per lipid was obtained from the lateral dimensions of the box. The curvature of the lipid patches was minimal so that this type of 
computation was accurate within the methods error. The lateral diffusion coefficient $D$ of lipids was obtained for each leaflet from a linear fit of the Mean Square Displacement of the phosphorus atoms (or corresponding coarse-grained bead) as a function of time, according to Einstein equation in two dimensions: ${ }^{110}\left\langle r^{2}\right\rangle=4 D t$. The fit was performed in the regions which showed a linear dependence, and the coefficients from the two leaflets were averaged. The COM movement of each leaflet was removed beforehand for control simulations involving only the membrane. The COM movement of the peptide assembly was instead removed when the capzip capsule or pentagonal unit was present and bound to the lipids. For atomistic simulations in presence of capzip pentagonal unit, the diffusion constant was also computed selectively on the lipids which, at the initial time, were within $1 \mathrm{~nm}, 2 \mathrm{~nm}$ or $3 \mathrm{~nm}$ from the protein, or in a region at distances larger than $3 \mathrm{~nm}$. Moreover, we monitored hydrogen bonds between capzip molecules and lipids.

For the umbrella sampling simulations of the capzip capsule penetrating the membrane, we derived the free energy profiles using the Weighted Histogram Analysis Method (WHAM) as implemented in GROMACS. ${ }^{111}$ The errors on the profiles were obtained through Bayesian bootstrapping, using 100 iterations.

\section{Results}

\section{Capzip in solution}

Atomistic simulations of the capzip capsule in solution show a structure going towards an equilibrated configuration within the force field applied, with a final arrangement slightly less regular than the initially imposed one. The atomistic RMSD (in purple in Fig. $3 \mathrm{~b}$ ) starts plateauing around $0.7 \mathrm{~nm}$ and the structure remains hollow. The system shrinks with respect to its initial configuration, as the gap between the two layers of the capsule tightens (final size $5.45 \mathrm{~nm}$, initial one $7 \mathrm{~nm}$ ), with an estimated wall thickness of $2.2 \mathrm{~nm}$. A similar trend is observed for MARTINI and SIRAH simulations, with RMSD of $1 \mathrm{~nm}$ and $1.5 \mathrm{~nm}$ (Fig. 3a), final size of $5.05 \mathrm{~nm}$ and $6.6 \mathrm{~nm}$ and wall thickness of $1.9 \mathrm{~nm}$ and $2.9 \mathrm{~nm}$, respectively. The SIRAH force field suggests more mobility of the arms and thus a larger rearrangement of the peptides, but the capsule still maintains a regular and hollow structure.

For the atomistic description, intermolecular contacts are mediated by hydrogen bonds (Fig. 4). In particular, the Tryptophan engages in $\mathrm{H}$-bonds with its backbone polar atoms, enforcing the $\beta$-sheet pairing hypothesised for the capzip antimicrobial arms. ${ }^{63}$ Arginine side chains engage with many other side chains as donors, and especially with glutamic acid, as expected from the juxtaposed positions they occupy in the $\beta$-sheet arrangement, and their opposite charges. This arrangement is reflected also in the coarsegrained descriptions by the proximity of the corresponding beads, attracted by electrostatic (not shown). Moreover, on average, 161 of the $180 \beta$-sheets keeping the capzip peptides a)

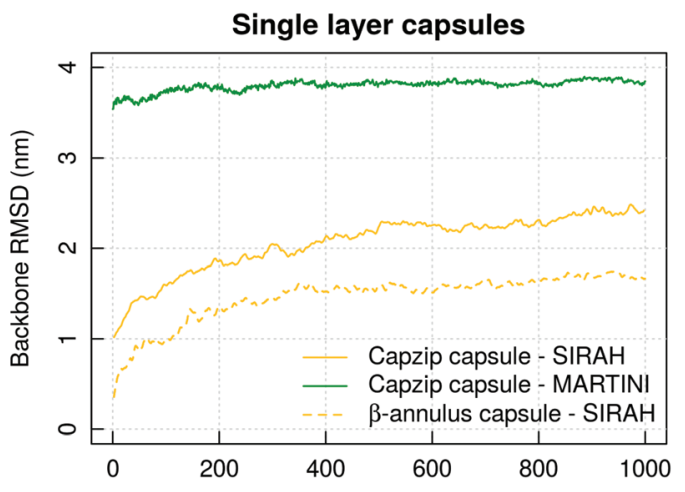

b)

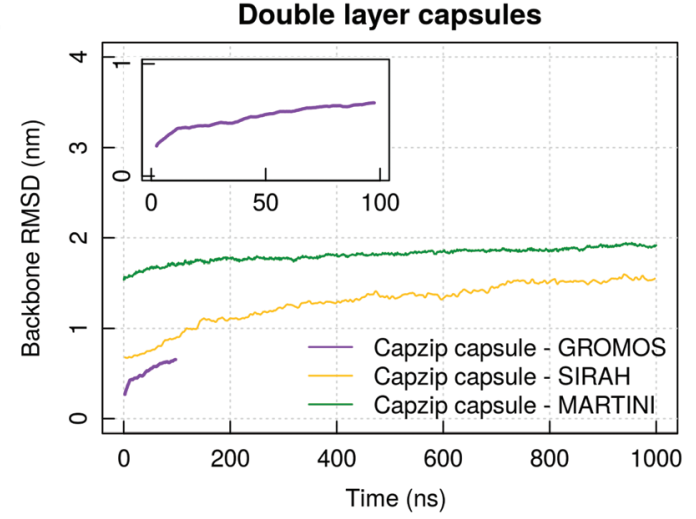

Fig. 3 Coarse-grained and atomistic simulations of single layer (a) and double layer capsules (b) formed by capzip or AM- $\beta$-annulus: RMSD computed on the protein backbone of the capsule. Results shown for replica 1. Inset: Enlarged plot from the atomistic GROMOS 54A8 simulations of the capzip capsule. Corresponding plots for $R_{\mathrm{g}}$ are shown in ESI Fig. $2 . \dagger$
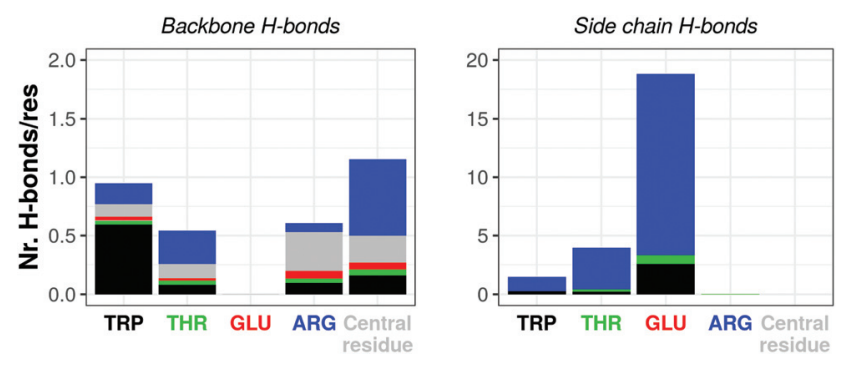

Fig. 4 Atomistic simulation of capzip capsule (replica 1): average number of hydrogen bonds per residue occurring between amino acids backbone (left) and side chains (right) in the last $50 \mathrm{~ns}$ of simulation (central residue refers to the moiety linking the antimicrobial arms - see Fig. 1). For each bar, the residue on the $x$-axis is the acceptor, and the bar is split by the identity of the donors. Corresponding plots for hydrogen bonds between side chain and backbone atoms are shown in ESI Fig. 3. $\dagger$

together in the capsule are maintained throughout the GROMOS 54A8 atomistic simulations, 140 in the MARTINI and 137 in the SIRAH forcefield (Fig. 5).

To prove that the bilayer is essential to prevent disassembly or deformation of the structure, we performed simulations of a monolayer capsule at the coarse-grained levels (specifically 

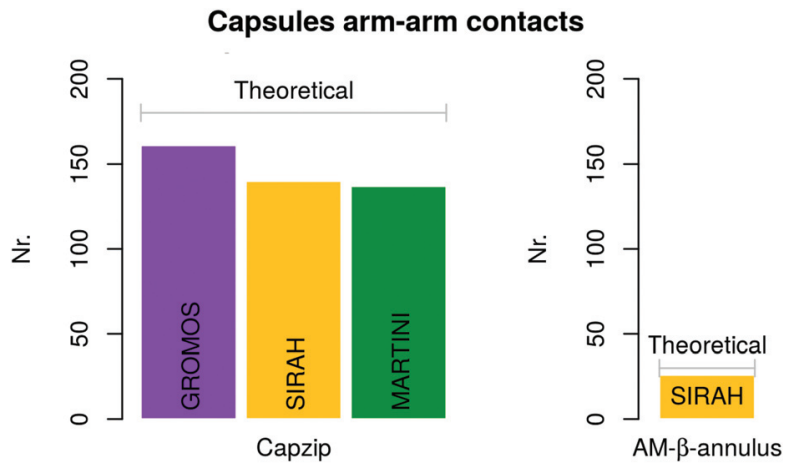

Fig. 5 Average number of conserved arm-arm contacts computed on the last 50 ns and 500 ns of atomistic and coarse-grained simulations, respectively. The grey bars indicate the expected number according to the geometry chosen, for both capzip and AM- $\beta$-annulus capsule.

taking the external layer of the capsule already simulated). The structure collapses toward its centre for both the MARTINI and SIRAH description, yielding a much higher RMSD than in the double layer case (3.8 nm versus $1.9 \mathrm{~nm}$ for MARTINI and $2.4 \mathrm{~nm}$ versus $1.4 \mathrm{~nm}$ for SIRAH, Fig. 3). Based on this, we concluded that the capzip capsules observed experimentally must have a non-monolayer structure to provide enough structural stability to the proposed geometrical assembly. This is consistent with cryo-EM images of capzip capsules which show a mutilayer structure. ${ }^{63}$

\section{Capzip-membrane interaction: atomistic simulations}

Comparing simulations of a bacterial model membrane (DLPC/DLPG 3:1, large patch of 740 lipids) with or without the capzip pentagonal unit in contact with it, no significant change is observed in the area per lipid ApL $\left(0.569(4) \mathrm{nm}^{2}\right)$. Similarly, a $20 \mathrm{mV} \mathrm{nm}^{-1}$ electric field (magnitude compatible with the transmembrane potential) does not affect the ApL in either configuration. The lateral diffusion varies between the replicas (between 0.3 and $0.5 \mu \mathrm{m}^{2} \mathrm{~s}^{-1}$ ) and for both lipids, but especially DLPG, the diffusion coefficient increases with the distance from the peptide (Fig. 6). This is due to the hydrogen bonds between both lipid species and the peptide, and net electrostatic interactions between positive arginines and negative DLPG lipids. We computed the former, for each amino acid type, and filtered for the ones present more than $50 \%$ of the simulation time (persistent bonds). When no field is applied, arginine residues promote 32 persistent bonds, 12 of which are with DLPG, although this lipid constitutes only $25 \%$ of the membrane. In a simulation with a $20 \mathrm{mV} \mathrm{nm}^{-1}$ electric field, they increase to 34,16 of which are with DLGP. Tryptophan and threonine residues promote fewer persisting bonds (10 and 8 for the simulation without field, and 9 and 11 for the one with electric field), with no particular preference for DLPG, and glutamate residues do not engage in any hydrogen bond. The central residue joining the arms does not form persistent hydrogen bonds with the lipids.

To observe the penetration of capzip through the membrane within the available simulation time, we performed atomistic

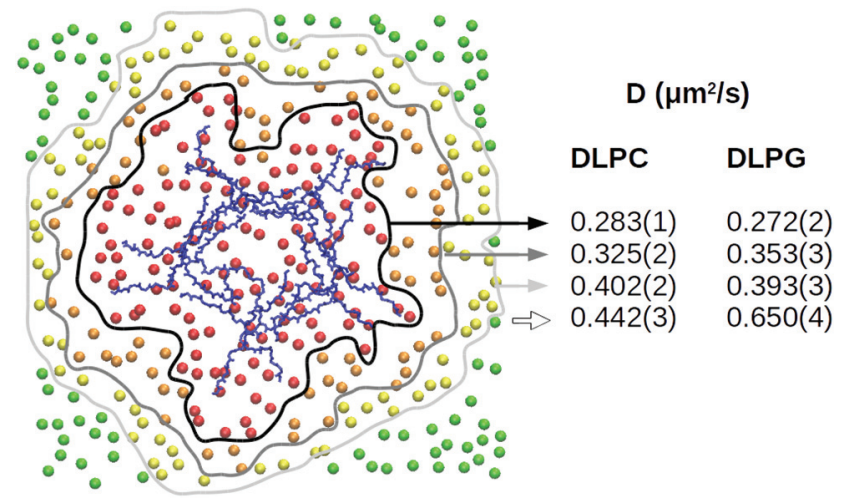

Fig. 6 Atomistic simulation of capzip pentagonal unit on model bacterial membrane (512 lipids): local lateral diffusion computed along 500 ns for DLPC and DLPG lipids which, at the initial time, are at a distance smaller than $1 \mathrm{~nm}$ (red), $2 \mathrm{~nm}$ (orange) or $3 \mathrm{~nm}$ (yellow) from the peptide or larger than $3 \mathrm{~nm}$ (green), centring the trajectory around the Protein COM (blue) [VMD software ${ }^{108}$ ].

electroporation simulations, by applying an increasing electric field on a 512 lipids patch (Fig. 7). Poration happened at the value of $130 \mathrm{mV} \mathrm{nm}^{-1}$ in presence of the peptide. This is confirmed by three replicas on both the 512 and 740 lipid patches, where the $130 \mathrm{mV} \mathrm{nm}^{-1}$ field was applied directly and poration happens within 20 to 75 ns of simulation in all the cases. Control simulations on a 512 lipid bacterial membrane without capzip show no poration at $130 \mathrm{mV} \mathrm{nm}^{-1}$ (600 ns simulations, 3 replicas). It was instead observed with a $140 \mathrm{mV} \mathrm{nm}^{-1}$ electric field after $150 \mathrm{~ns}$ and $154 \mathrm{~ns}$, while a third replica presented a curved but still intact membrane after $200 \mathrm{~ns}$.

The application of an electric field to the system of capzip capsule-bacterial membrane accelerates the bilayer disruption process, as reported previously. ${ }^{112}$ This is due to perturbations

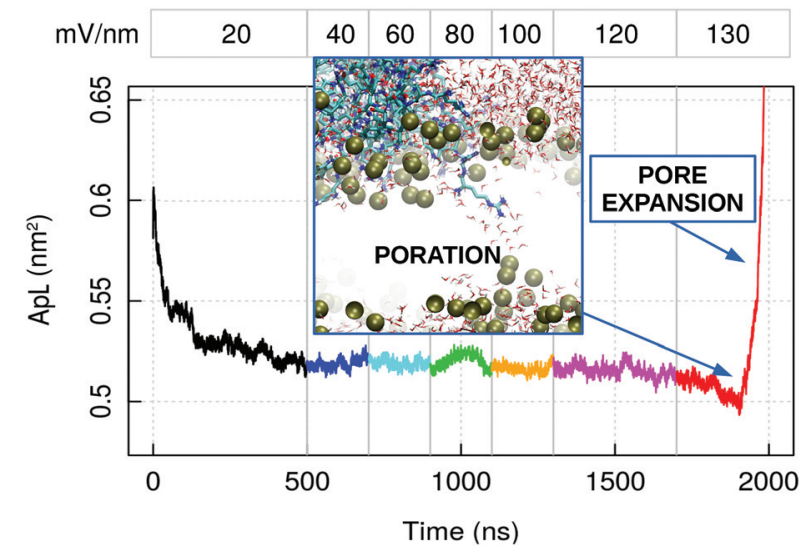

Fig. 7 Atomistic simulation of capzip pentagonal unit on model bacterial membrane (512 lipids) with increasing applied external electric field: average area per lipid (ApL). The numbers at the top indicate the magnitude of the field in $\mathrm{mV} \mathrm{nm}^{-1}$. The inset shows the poration event: Lipid boundaries in surface representation, peptides and water molecules in bond representation [VMD software ${ }^{108}$ ]. The increase in ApL after pore formation is due to the pore expansion. 
caused to the membraneous arrangement by the inserting peptide through charged and hydrogen bond interactions mentioned before. These promote charged arginine residue insertion and initiate water penetration in the hydrophobic core of the membrane. Similar initiation processes have been observed for arginine rich cell penetrating peptides in experiments ${ }^{113,114}$ and MD simulations. ${ }^{114,115}$

\section{Capzip-membrane interactions: coarse-grained simulations}

Coarse-grained simulations allow to model the full capsule interacting with the membrane. Simulations with MARTINI (standard water model) proved the binding of capzip capsule to the bacterial model membrane, but not to the mammal (in the $10 \mu$ s simulated). In both runs, the peptide gets in contact with the bacterial membrane after about $2 \mu$ s and remains membrane-bound, diffusing laterally and producing an increasingly high curvature on the bilayer (Fig. 8, top). No poration is observed, probably because longer time scales are needed to simulate it.

The binding is driven by charge-charge interactions, and the presence of the capsule impacts strongly on the diffusion and distribution of the lipids. The lateral diffusion coefficient drops from 74.28(1) to 70.51(1) $\mu \mathrm{m}^{2} \mathrm{~s}^{-1}$ for DLPC, and from 73.34(2) to $62.59(2) \mu \mathrm{m}^{2} \mathrm{~s}^{-1}$ for DLPG. The larger effect on DLPG is due to its negative net charge and a strong attraction to the positively charged peptide. This is confirmed by the
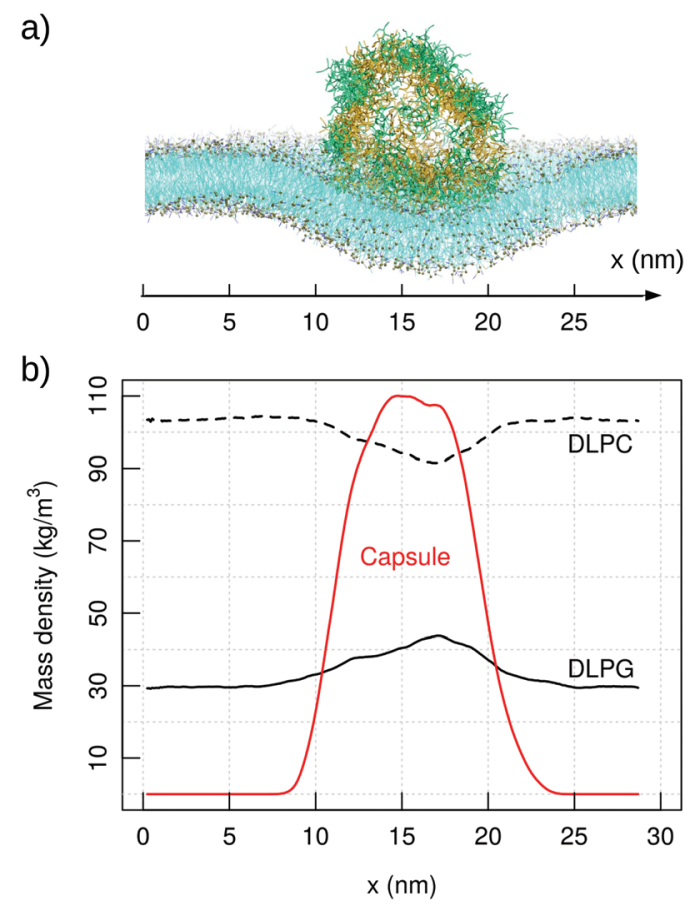

Fig. 8 MARTINI simulation of capzip capsule on bacterial model membrane $(10 \mu \mathrm{s})$ : (a) snapshot of the final configuration (capsule in stick representation, outer and inner layer in green and yellow respectively; lipids in line representation and phosphate beads in golden van der Waals VMD software ${ }^{108}$ ); (b) corresponding mass density of capzip, DLPC and DLPG along the $x$-axis, averaged on the last $\mu$ s of simulation.
RDF of the capzip amino acids around different lipids moieties, as the strongest signal comes from the Arg residue around the DLPG phosphate groups, and is less so from the DLPC phosphate moieties (ESI Fig. 6†). As a consequence, DLPG lipids are enriched nearby the capsule (Fig. 8), at the expense of DLPC molecules. This enrichment can be observed at the coarse-grained level only, as the lipid patch is large enough, and the time simulated sufficient, to allow a collective rearrangement of the membrane components.

The adoption of Polar water ${ }^{103}$ alone (without applied electric field) sped up considerably the binding of the capsule to the membrane (within the first $50 \mathrm{~ns}$, as opposed to $2 \mu \mathrm{s}$ of the standard water simulation). Then, in a 500 ns simulation under the action of a $20 \mathrm{mV} \mathrm{nm}{ }^{-1}$ electric field, the curvature of the membrane rapidly increased, and after switching to a $40 \mathrm{mV} \mathrm{nm}{ }^{-1}$ field the membrane porated within $200 \mathrm{~ns}$. Analogous simulations on the membrane alone did not trigger poration in $500 \mathrm{~ns}\left(40 \mathrm{mV} \mathrm{nm}{ }^{-1}\right)$. Continuing the simulation of the porated membrane $\left(40 \mathrm{mV} \mathrm{nm}{ }^{-1}\right)$, we observe the capsule penetrating within $10 \mathrm{~ns}$. The lipids do not seal around the capsule, allowing the passage of water and ions, which is consistent with our findings for the atomistic simulations. During the membrane penetration process, the capsule deforms and partially opens. Longer simulations might lead to poration with a lower field strength, as the initiation of a pore is a stochastic event. Nevertheless, the simulations set an important comparison with the model mammalian membrane, as for the latter no binding was observed even with an applied electric field.

Simulations of the phospholipid mixture DLPC/DLPG elucidates why the capzip capsule is active on in vitro model lipid bilayers and reproduces the experimental conditions with which capzip was developed. ${ }^{18,63}$ One could enquire how this activity can be tested with models of the Gram negative outer membrane. We performed two simulations of the capzip capsule on the LPS membrane, for a total of $2 \mu \mathrm{s}$. Starting with the capsule at $1 \mathrm{~nm}$ distance from the LPS membrane, the capsule attaches to it after $270 \mathrm{~ns}$, attracted by the negative charge of the LPS molecules. The membrane is thicker and thus less flexible than the previous DLPC/DLPG model: while in that case the charge-charge interaction forced the membrane to curve and partially wrap the capsule, on LPS this effect is smaller. On the contrary the capsule quickly opens to increase the interactions with the LPS molecules after the attachment, rearranging itself in the following $300 \mathrm{~ns}$, after which the configuration is kept stable (ESI Fig. 10†). The peptide residues lying on the surface penetrate down to the region of calcium ions, but do not cross it in the simulation time. Arginine residues are also in this case the ones penetrating deeper. This is confirmed by the simulation starting with the capsule in contact with the membrane.

As penetration events are slow, longer simulations might indicate further rearrangement of the peptides. Additionally, the properties of the membrane at the interface are significantly influenced by the presence of high concentration of calcium ions ${ }^{116}$ and therefore the mechanism of adhesion and 
penetration of the capsule maybe severely altered with respect to the DLPC/DLPG membrane. More accurate studies varying calcium concentration and fine tuning the interactions with the highly charged capsule and lipids are necessary to elucidate this. Of note, the capsule simulated has a size of $15 \mathrm{~nm}$, while the experiments detect capsules of bigger sizes as well, ${ }^{18,63}$ and a larger localised concentration of capzip molecules would be more effective in perturbing the membrane.

To further clarify the difference in the mechanisms of interaction of the capzip peptide between the bacterial and mammal model membrane, and to speed up the process of capzip capsule membrane passage, we pulled the capsule through the DLPC/DLPG and the DLPC model membranes. We reverted back to the simpler bacterial model membrane (mimicking the inner membrane) as a simpler and more easily interpretable system. The PMF profile was computed using an umbrella sampling procedure (Fig. 9). In the mammalian model, the membrane constitutes a high energy barrier to the approach and penetration of the capsule, and the PMF rises consistently while the capsule-membrane distance is decreasing. To be noticed that the distance reported in Fig. 9 is measured as the $z$ position of the centre of mass of the capsule minus the $z$ position of the centre of mass of an outer disc of lipids, i.e. lipids which are not within a $12 \mathrm{~nm}$ radius from the $z$-projection of the capsule COM on the membrane. Poration happens at distance larger than $0 \mathrm{~nm}$ due to the size of the capsule. After the disruption, the membrane quickly rearranges around the capsule. As umbrella sampling should be performed in near to equilibrium conditions for each window, we did not evaluate the PMF after the poration event.

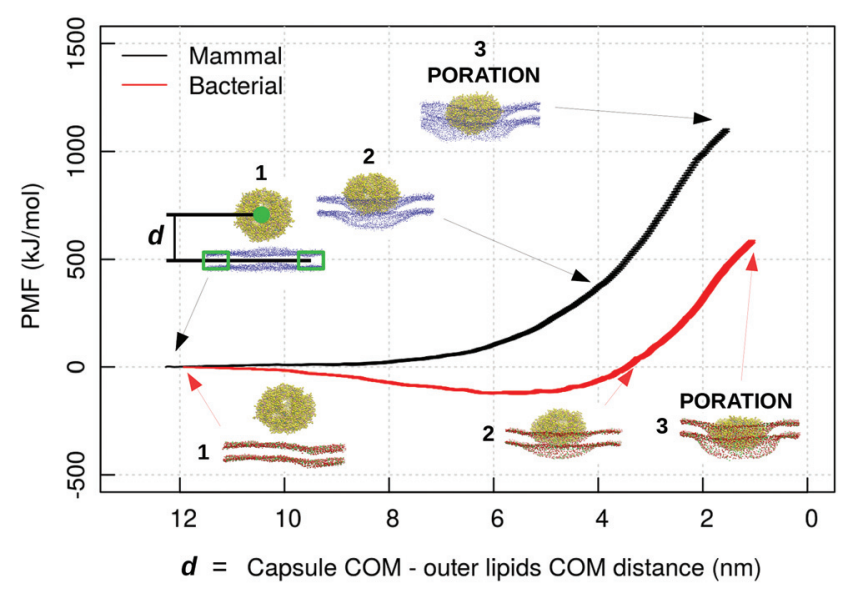

Fig. 9 PMF profile of a capzip capsule penetrating a mammal (black) and bacterial (red) model membrane (MARTINI simulations), in function of the distance $d$ between capzip capsule centre of mass and the centre of mass of the outer ring of lipids not involved in the pulling groups (lipids which are not within a $12 \mathrm{~nm}$ radius from the $z$-projection of the capsule COM on the membrane), which represent the unperturbed membrane. The snapshots show different stages of the penetration process; peptide and phosphate beads of lipids in van der Waals representation (blue for DLPC in the mammal membrane; red and green for DLPC and DLPG in the bacterial one) [VMD software ${ }^{108}$ ].
Nevertheless the simulation brings some useful insight. Contrary to the mammal case, for the bacterial membrane the PMF decreases during the capsule approach (of about $100 \mathrm{~kJ}$ $\mathrm{mol}^{-1}$ ), and increases only afterwards. The first effect is promoted by the negatively charged lipids which attract the positive capsule. This proves once more that the bacterial membrane has a stronger affinity for the capzip capsule and therefore their poration and disruption action is more pronounced for these systems, and consequently the antimicrobial function is more effectively exerted.

As stated in the methods, while the combination of atomistic and MARTINI force fields was selected for the exploratory analysis, the SIRAH force field, of intermediate granularity between the two, is suitable to pursue further investigation on the $\beta$-annulus interaction with the membrane. Moreover, the $\beta$-annulus has a well defined secondary structure in its core and the SIRAH set up allows to simulate the molecule without recurring to constraints to maintain its secondary structure like in MARTINI's approach. To confirm the suitability of the force field we repeated the simulations of capzip capsule on the bacterial membrane with the SIRAH parameters. The capsule attaches to the membrane within the first $100 \mathrm{~ns}$ and deforms it but no poration is observed in the $10 \mu \mathrm{s}$ simulated, consistent with the MARTINI simulations. The diffusion of the charged lipids (POPS in this case) is similar to the one of the zwitterionic POPC, being respectively 16.2. and $16.6 \mu \mathrm{m}^{2} \mathrm{~s}^{-1}$ and no enrichment of POPS nearby the capsule is observed. In the MARTINI simulations, the negatively charged lipid was recruited within the same amount of simulation time: the difference with the SIRAH force field is likely due to the different parametrisations.

\section{Functionalised $\beta$-annulus simulations}

Simulations of the AM- $\beta$-annulus capsule in solution show that the capsule equilibrates (with RMSD of $1.7 \mathrm{~nm}$ ) with a radius of gyration of $4.5 \mathrm{~nm}$ (Fig. 3 and ESI Fig. $1 \dagger$ ). Similarly to the capzip capsule, it shrinks during the simulation to achieve its equilibrium size. Out of the 30 pre-imposed armarm pairs, 26 are maintained during the simulation, proving that the initial geometry is well conserved.

Simulations of the AM- $\beta$-annulus capsule on the bacterial membrane show attachment to the lipids within $1 \mu \mathrm{s}$ of the simulation without electric field. This is compatible with what observed for capzip, however with the introduction of a small electric field ( $4 \mathrm{mV} \mathrm{nm}{ }^{-1}$ ), a small pore is forming within $1.5 \mu \mathrm{s}$, contrary to capzip, for which poration is not observed in the $10 \mu \mathrm{s}$ simulated (Fig. 10). Some portions of the capsule reach the core of the membrane (Fig. 11). The presence of the AM- $\beta$-annulus capsule on the membrane does not affect the lateral diffusion of lipids, which remains $14.2 \mu \mathrm{m}^{2} \mathrm{~s}^{-1}$ for POPC and $15.9 \mu \mathrm{m}^{2} \mathrm{~s}^{-1}$ for POPS, nor induces any recruitment of the negatively charged POPS.

In investigating the reasons for the pore formation, we computed the Radial Distribution Function of any of the amino acid type forming the AM- $\beta$-annulus sequence around typical moieties of the lipids heads, in order to identify key peptide- 


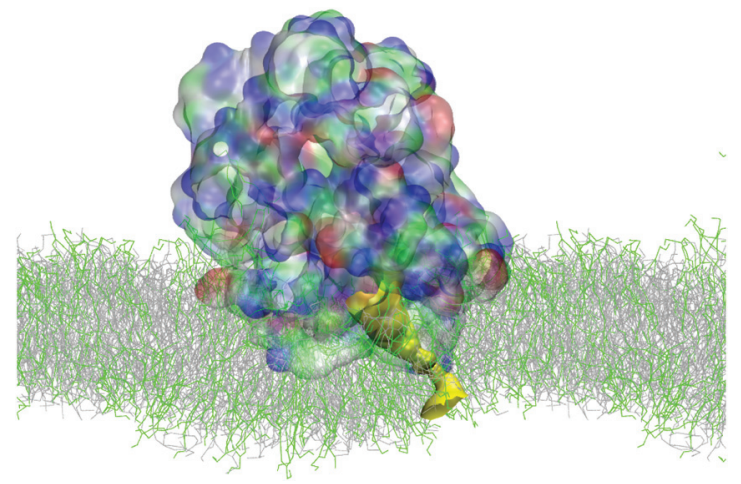

Fig. 10 SIRAH simulation of AM- $\beta$-annulus capsule on bacterial model membrane: snapshot of poration due to the combined action of the capsule and a $4 \mathrm{mV} \mathrm{nm}^{-1}$ electric field. Capsule in surface representation, color coded by amino acid type (blue positive, red negative, green polar, white hydrophobic). Pore highlighted in yellow surface. Lipids in grey (POPC) and green (POPS) lines [VMD software ${ }^{108}$ ].

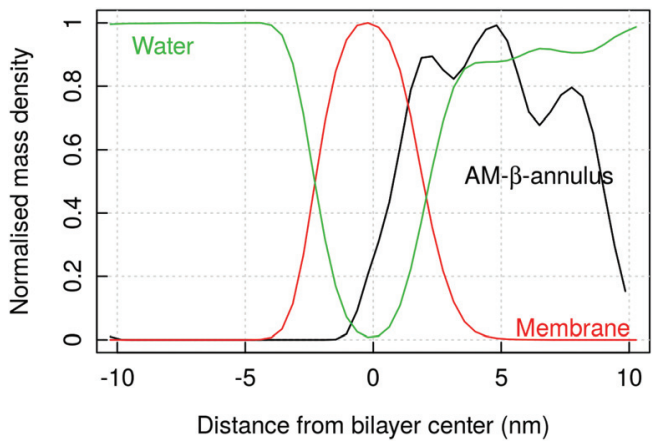

Fig. 11 SIRAH simulation of $A M-\beta$-annulus capsule on a bacterial model membrane: mass density of the peptide, lipids and water, normalised (maximum density value is 1 for each component). The oscillations in the AM- $\beta$-annulus profile reflect the presence of holes in the capsule architecture.

lipid interactions. It emerges a strong interaction of the positive POPC choline head group with the negative glutamic acid residue, and of the negative phosphate groups with lysine and arginine residues. Additionally, lysine interacts with the L-serine POPS head group (Fig. 12, similar plots for all the other residues are shown in ESI Fig. 8 and $9 \dagger$ ). In the case of the capzip capsule, the tryptophan interacts very weakly with the DLPC glycerol and slightly more with the DLPG one (ESI Fig. 6 and $7 \dagger$ ). For the AM- $\beta$-annulus capsule instead the hydrophobic residues are interacting with the lipids.

This is compatible with the values of Surface Accessible Solvent Area $\left(Q_{\mathrm{SASA}}\right.$, normalised by the nominal experimental value) computed for positive, negative, polar and hydrophobic amino acids on the atomistic structures of capzip and AM$\beta$-annulus capsules (ESI Table $1 \dagger$ ). The positive amino acids $Q_{\mathrm{SASA}}$ is higher for the capzip capsule (more exposed positive residues), and the hydrophobic one is lower (less exposed), leading to a faster attachment of the capzip capsule to the membrane (100 ns versus $500 \mathrm{~ns})$ driven by a charge recognition
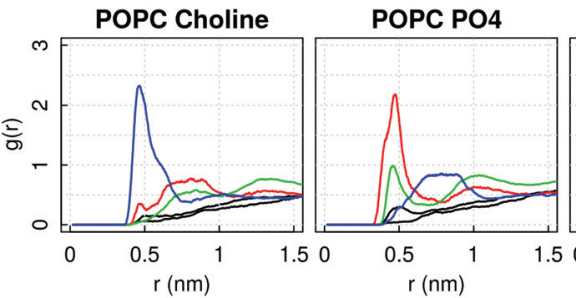

POPC Glycerol

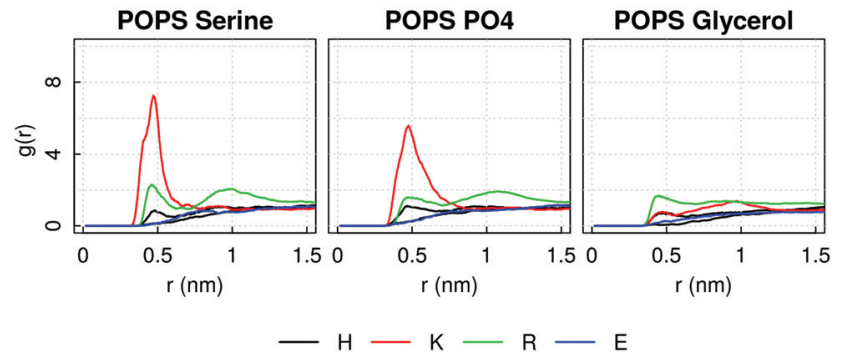

Fig. 12 SIRAH simulation of AM- $\beta$-annulus capsule on a bacterial model membrane: Radial distribution function of AM- $\beta$-annulus charged amino acids around typical moieties of lipids heads, averaged over the time simulated after the capsule binding.

mechanism. On the contrary, in the AM- $\beta$-annulus capsule, the more exposed hydrophobic residues facilitate the insertion process, with their propensity to segregate from water.

The difference in hydrophobic residues exposition is due to the double layer structure of the capzip capsule, but also on the higher content of hydrophobic residues in the AM$\beta$-annulus. Thus features from the core of this construct are contributing to the antimicrobial activity together with the RRWTWE sequence itself.

\section{Conclusions}

The investigations performed proved the power of the use of combined protein design and molecular simulations with force fields of different granularity to elucidate details of nanoscale systems with antimicrobial activity. We explored potential arrangements in pre-assembled nanocapsule systems of a specific antimicrobial sequence from Lactoferrin and studied their stability and their interaction with model membranes. The two systems reported here were selected for different reasons: for the capzip system we had a number of supporting experiments suggesting that our design in a double layered capsule is correct; we wanted to explore in detail the interaction with mammals $v s$. non mammals model membranes to clarify the efficacy of the capzip system. For the AM- $\beta$-annulus system we have proposed a designed nanocapsule with self assembling elements: (a) a peptide element that is experimentally proven to self-assemble in a triskelion geometry suitable for a nanocapsule; ${ }^{74-79}$ and (b) the antimicrobial sequence used for the capzip system as antimicrobial moiety. ${ }^{18,63}$ This original design has been investigated for its stability in solution and interaction with a model membrane in silico. 
When investigating the assembly process of capzip peptides, the use of atomistic simulations gave insights into the role of specific amino acids in the pairing and assembly of the many copies of the molecule, the role of the 'arms' and the precise indentation of the residues forming the $\beta$-sheet elements necessary to hold the scaffold of the capsule.

Coarse-grained simulations proved extremely useful in the evaluation of the stability of the pre-assembled structures on the long time scale. Additionally, we showed here that simplified models were sufficient to evaluate processes like the absorption on different types of membranes. Overall, we were able to conclude that the ability of capzip to form capsules lays not only in the scheme of opposite charges that it hosts along its arms, but also in the presence of many hydrophobic residues, which favour the assembly with attractive hydrophobic forces. Thus, the proposed structure contains a bilayer arrangement of the molecules, which is demonstrated to be more stable than a monolayer one, and is consistent with the more recent experimental findings showing multilayer capsules. ${ }^{63}$

Coarse-grained simulations were also able to clarify the interaction of capzip with the membrane. Capzip has a propensity to bind to negatively charged membranes, which are a simplified model for the bacterial inner membrane, but not to zwitterionic lipids, which model a mammalian membrane. Moreover, on the bacterial membrane, the buckyball recruits negatively charged lipids in its proximity, reducing their diffusion. Atomistic simulations complemented these findings showing the details of such interaction: on a bacterial membrane, capzip interacts with the lipids forming many hydrogen bonds and inserting the Arginine side chains deep in the phosphate region of the lipids. Consequently, the lipids around the peptide are slowed down in their diffusion on the measured time scale of atomistic simulations.

Finally, simulations with an external electric field applied (within physiological ranges) to the system showed that the peptide bound to the bacterial membrane promotes poration at values of the field which would not cause electroporation on the membrane alone.

The above results integrate the ones from pulling experiments, which show a favourable attraction between capzip and a model bacterial membrane, and a lower energy barrier for poration in the bacterial versus the mammal membrane case. This gives a more complete picture on the characteristics of this capzip, in agreement with the experimentally proven selectivity but non-toxicity of the peptide. ${ }^{18,63}$

The results on the AM- $\beta$-annulus system prove the possibility of designing AM nanocapsules with self-assembling elements that can impose a specific geometry to the capsule. We hope that the proposed design will stimulate experimental studies on the stability and organisation of the assembly in monolayer capsules and on their antimicrobial activity.

The outcome of the research presented here can be leveraged for future design strategies as it highlights the crucial and necessary components for the assembly, as well as the ones where there is scope for improvement or modification. Future investigations will tackle this problem, unifying again the experimental and computational settings to help a fast screening of newly generated compounds.

\section{Conflicts of interest}

There are no conflicts to declare.

\section{Acknowledgements}

I. M. acknowledges funding by the Engineering and Physical Sciences Research Council (EPSRC) through the Centre for Doctoral Training "Cross Disciplinary Approaches to NonEquilibrium Systems" (CANES, Grant No. EP/L015854/ 1). C. H. B. C. acknowledges funding from CAPES - Proc. $n$ 88881.197985/2018-01. This project made use of the following resources: computer time on ARCHER granted via the UK High-End Computing Consortium for Biomolecular Simulation, HECBioSim (http://hecbiosim.ac.uk), supported by EPSRC (grant no. EP/R029407/1); resources provided by the Cambridge Service for Data Driven Discovery (CSD3) operated by the University of Cambridge Research Computing Service (http://www.csd3.cam.ac.uk/), provided by Dell EMC and Intel using Tier-2 funding from the Engineering and Physical Sciences Research Council (capital grant EP/P020259/1), and DiRAC funding from the Science and Technology Facilities Council (http://www.dirac.ac.uk).

\section{References}

1 P. Mantravadi, K. Kalesh, R. Dobson, A. Hudson and A. Parthasarathy, Antibiotics, 2019, 8, 8.

2 L. T. Nguyen, E. F. Haney and H. J. Vogel, Trends Biotechnol., 2011, 29, 464-472.

3 A. Ebbensgaard, H. Mordhorst, M. T. Overgaard, C. G. Nielsen, F. M. Aarestrup and E. B. Hansen, PLoS One, 2015, 10, e0144611.

4 T. Ebenhan, O. Gheysens, H. G. Kruger, J. R. Zeevaart and M. M. Sathekge, BioMed Res. Int., 2014, 867381.

5 M. Mahlapuu, J. Håkansson, L. Ringstad and C. Björn, Front. Cell. Infect. Microbiol., 2016, 6, 194.

6 A. Peschel and H.-G. Sahl, Nat. Rev. Microbiol., 2006, 4, 529-536.

7 A. Lewies, L. H. Du Plessis and J. F. Wentzel, Probiotics Antimicrob. Proteins, 2019, 11, 370-381.

$8 \mathrm{~K}$. Hilpert, R. Volkmer-Engert, T. Walter and R. E. W. Hancock, Nat. Biotechnol., 2005, 23, 1008-1012.

9 D. Migoń, M. Jaśkiewicz, D. Neubauer, M. Bauer, E. Sikorska, E. Kamysz and W. Kamysz, Probiotics Antimicrob. Proteins, 2018, 1-13.

10 S. Liu, J. Bao, X. Lao and H. Zheng, Sci. Rep., 2018, 8, 11189.

11 Z. Jiang, A. I. Vasil, L. Gera, M. L. Vasil and R. S. Hodges, Chem. Biol. Drug Des., 2011, 77, 225-240. 
12 C. D. Fjell, J. A. Hiss, R. E. W. Hancock and G. Schneider, Nat. Rev. Drug Discovery, 2011, 11, 37.

13 V. V. Kleandrova, J. M. Ruso, A. Speck-Planche and M. N. Dias Soeiro Cordeiro, ACS Comb. Sci., 2016, 18, 490498.

14 D. Veltri, U. Kamath and A. Shehu, Bioinformatics, 2018, 34, 2740-2747.

15 F. Cipcigan, A. P. Carrieri, E. O. Pyzer-Knapp, R. Krishna, Y.-W. Hsiao, M. Winn, M. G. Ryadnov, C. Edge, G. Martyna and J. Crain, J. Chem. Phys., 2018, 148, 241744.

16 H. Khandelia and Y. N. Kaznessis, Peptides, 2005, 26, 2037-2049.

17 A. Farrotti, P. Conflitti, S. Srivastava, J. Ghosh, A. Palleschi, L. Stella, G. Bocchinfuso, A. Farrotti, P. Conflitti, S. Srivastava, J. K. Ghosh, A. Palleschi, L. Stella and G. Bocchinfuso, Molecules, 2017, 22, 1235.

18 V. Castelletto, E. de Santis, H. Alkassem, B. Lamarre, J. E. Noble, S. Ray, A. Bella, J. R. Burns, B. W. Hoogenboom, M. G. Ryadnov, J. Vandesompele and C. T. Wittwer, Chem. Sci., 2016, 7, 1707-1711.

19 H. Sun, Y. Hong, Y. Xi, Y. Zou, J. Gao and J. Du, Biomacromolecules, 2018, 19, 1701-1720.

20 F. Cao, L. Mei, G. Zhu, M. Song and X. Zhang, RSC Adv., 2019, 9, 30803-30808.

21 C. Morris, S. J. Glennie, H. S. Lam, H. E. Baum, D. Kandage, N. A. Williams, D. J. Morgan, D. N. Woolfson and A. D. Davidson, Adv. Funct. Mater., 2019, 29, 1807357.

22 G. Manzo, P. M. Ferguson, C. K. Hind, M. Clifford, V. B. Gustilo, H. Ali, S. S. Bansal, T. T. Bui, A. F. Drake, R. A. Atkinson, J. M. Sutton, C. D. Lorenz, D. A. Phoenix and A. J. Mason, Sci. Rep., 2019, 9, 10934.

23 G. Manzo, P. M. Ferguson, V. B. Gustilo, C. K. Hind, M. Clifford, T. T. Bui, A. F. Drake, R. A. Atkinson, J. M. Sutton, G. Batoni, C. D. Lorenz, D. A. Phoenix and A. J. Mason, Sci. Rep., 2019, 9, 1385.

24 S. B. T. Amos, L. S. Vermeer, P. M. Ferguson, J. Kozlowska, M. Davy, T. T. Bui, A. F. Drake, C. D. Lorenz and A. J. Mason, Sci. Rep., 2016, 6, 1-13.

25 H. Leontiadou, A. E. Mark and S.-J. Marrink, J. Am. Chem. Soc., 2006, 128, 12156-12161.

26 J. P. Ulmschneider, Biophys. J., 2017, 113, 73-81.

27 Y. Wang, C. H. Chen, D. Hu, M. B. Ulmschneider and J. P. Ulmschneider, Nat. Commun., 2016, 7, 13535.

28 D. Sun, J. Forsman and C. E. Woodward, J. Phys. Chem. B, 2015, 119, 14413-14420.

29 L. Zhao, Z. Cao, Y. Bian, G. Hu, J. Wang and Y. Zhou, Int. J. Mol. Sci., 2018, 19, 1186.

30 Y. Wang, D. E. Schlamadinger, J. E. Kim and J. A. McCammon, Biochim. Biophys. Acta, Biomembr., 2012, 1818, 1402-1409.

31 R. Lipkin, A. Pino-Angeles and T. Lazaridis, J. Phys. Chem. B, 2017, 121, 9126-9140.

32 G. E. Balatti, E. E. Ambroggio, G. D. Fidelio, M. F. Martini and M. Pickholz, Molecules, 2017, 22(10), 1775-1792.

33 D. Tieleman, Biophys. J., 2017, 113, 1-3.
34 D. Jefferies and S. Khalid, J. Mol. Biol., 2020, 432, 12511264.

35 C. Chen, C. G. Starr, E. P. Troendle, G. Wiedman, W. C. Wimley, J. P. Ulmschneider and M. B. Ulmschneider, J. Am. Chem. Soc., 2019, 141, 48394848.

36 Y. Zhao, W. Yang, D. Wang, J. Wang, Z. Li, X. Hu, S. King, S. Rogers, J. R. Lu and H. Xu, Small, 2018, 14, 1703216.

37 E. Glukhov, M. Stark, L. L. Burrows and C. M. Deber, J. Biol. Chem., 2005, 280, 33960-33967.

38 M. Tang and M. Hong, Mol. BioSyst., 2009, 5, 317.

39 D. Poger, W. F. Van Gunsteren and A. E. Mark, J. Comput. Chem., 2010, 31, 1117-1125.

40 S.-W. Chiu, M. Clark, V. Balaji, S. Subramaniam, H. L. Scott and E. Jakobsson, Biophys. J., 1995, 69, 12301245.

41 T. J. Piggot, D. A. Holdbrook and S. Khalid, J. Phys. Chem. $B, 2011,115,13381-13388$.

42 I. Marzuoli, C. Margreitter and F. Fraternali, J. Chem. Theory Comput., 2019, 15, 5175-5193.

43 A. Kukol, J. Chem. Theory Comput., 2009, 5, 615-626.

44 J. C. Gumbart, M. Beeby, G. J. Jensen and B. Roux, PLoS Comput. Biol., 2014, 10, 1-10.

45 M. L. Ortiz-Suarez, F. Samsudin, T. J. Piggot, P. J. Bond and S. Khalid, Biophys. J., 2016, 111, 1692-1702.

46 K. N. Kirschner, R. D. Lins, A. Maass and T. A. Soares, J. Chem. Theory Comput., 2012, 8, 4719-4731.

47 T. J. Piggot, D. A. Holdbrook and S. Khalid, Biochim. Biophys. Acta, Biomembr., 2013, 1828, 284-293.

48 P.-C. Hsu, D. Jefferies and S. Khalid, J. Phys. Chem. B, 2016, 120, 11170-11179.

49 T. J. Silhavy, D. Kahne and S. Walker, Cold Spring Harbor Perspect. Biol., 2010, 2, a000414-a000414.

50 D. P. Tieleman, BMC Biochem., 2004, 5, 10.

51 R. A. Böckmann, B. L. de Groot, S. Kakorin, E. Neumann and H. Grubmüller, Biophys. J., 2008, 95, 1837-1850.

52 J. Lin and A. Alexander-Katz, ACS Nano, 2013, 7, 1079910808.

53 K. Shimizu, H. Nakamura and S. Watano, Nanoscale, 2016, 8, 11897-11906.

54 H. Nakamura, K. Sezawa, M. Hata, S. Ohsaki and S. Watano, Phys. Chem. Chem. Phys., 2019, 21, 18830-18838.

55 J. T. Sengel and M. I. Wallace, Philos. Trans. R. Soc., B, 2017, 372, 20160227.

56 L. Yang, T. A. Harroun, T. M. Weiss, L. Ding and H. W. Huang, Biophys. J., 2001, 81, 1475-1485.

57 K. J. Hallock, D.-K. Lee and A. Ramamoorthy, Biophys. J., 2003, 84, 3052-3060.

58 A. Spaar, C. Münster and T. Salditt, Biophys. J., 2004, 87, 396-407.

59 J. T. Wiemann, Z. Shen, H. Ye, Y. Li and Y. Yu, Nanoscale, 2020, 12, 20326-20336.

60 F. Samsudin and S. Khalid, J. Phys. Chem. B, 2019, 123, 2824-2832.

61 A. T. Asmar and J.-F. Collet, FEMS Microbiol. Lett., 2018, 365, fny199. 
62 S. Khalid, T. J. Piggot and F. Samsudin, Acc. Chem. Res., 2019, 52, 180-188.

63 I. E. Kepiro, I. Marzuoli, K. Hammond, X. Ba, H. Lewis, M. Shaw, S. B. Gunnoo, E. De Santis, U. Łapińska, S. Pagliara, M. A. Holmes, C. D. Lorenz, B. W. Hoogenboom, F. Fraternali and M. G. Ryadnov, ACS Nano, 2019, 14, 1609-1622.

64 P. M. Hwang, N. Zhou, X. Shan, C. H. Arrowsmith and H. J. Vogel, Biochemistry, 1998, 37, 4288-4298.

65 D. J. Schibli, P. M. Hwang and H. J. Vogel, FEBS Lett., 1999, 446, 213-217.

66 A. G. Cochran, N. J. Skelton and M. A. Starovasnik, Proc. Natl. Acad. Sci. U. S. A., 2001, 98, 5578-5583.

67 M. Ashrafuzzaman, C. Y. Tseng and J. A. Tuszynski, J. Mol. Graphics Modell., 2020, 95, 107502.

68 W. Wu, S. C. Hsiao, Z. M. Carrico and M. B. Francis, Angew. Chem., Int. Ed., 2009, 48, 9493-9497.

69 L. Schoonen and J. C. M. van Hest, Nanoscale, 2014, 6, 7124-7141.

70 R. F. Silva, D. R. Araújo, E. R. Silva, R. A. Ando and W. A. Alves, Langmuir, 2013, 29, 10205-10212.

71 T. O. Yeates, Nature, 2019, 569, 340-342.

72 A. D. Malay, N. Miyazaki, A. Biela, S. Chakraborti, K. Majsterkiewicz, I. Stupka, C. S. Kaplan, A. Kowalczyk, B. M. A. G. Piette, G. K. A. Hochberg, D. Wu, T. P. Wrobel, A. Fineberg, M. S. Kushwah, M. Kelemen, P. Vavpetič, P. Pelicon, P. Kukura, J. L. P. Benesch, K. Iwasaki and J. G. Heddle, Nature, 2019, 569, 438-442.

73 P. Hopper, S. C. Harrison and R. T. Sauer, J. Mol. Biol., 1984, 177, 701-713.

74 K. Matsuura, K. Watanabe, T. Matsuzaki, K. Sakurai and N. Kimizuka, Angew. Chem., Int. Ed., 2010, 49, 9662-9665.

75 S. Fujita and K. Matsuura, Nanomaterials, 2014, 4, 778-791.

76 K. Matsuura, G. Ueno and S. Fujita, Polym. J., 2015, 47, 146-151.

77 Y. Nakamura, S. Yamada, S. Nishikawa and K. Matsuura, J. Pept. Sci., 2017, 23, 636-643.

78 K. Matsuura, Y. Mizuguchi and N. Kimizuka, Biopolymers, 2016, 106, 470-475.

79 K. Matsuura, T. Nakamura, K. Watanabe, T. Noguchi, K. Minamihata, N. Kamiya and N. Kimizuka, Org. Biomol. Chem., 2016, 14, 7869-7874.

80 C. Oostenbrink, T. A. Soares, N. F. A. van der Vegt and W. F. van Gunsteren, Eur. Biophys. J., 2005, 34, 273-284.

81 M. M. Reif, M. Winger and C. Oostenbrink, J. Chem. Theory Comput., 2013, 9, 1247-1264.

82 S.-J. Marrink, H. J. Risselada, S. Yefimov, D. P. Tieleman and A. H. De Vries, J. Phys. Chem. B, 2007, 111, 7812-7824.

83 L. Monticelli, S. K. Kandasamy, X. Periole, R. G. Larson, D. P. Tieleman and S.-J. Marrink, J. Chem. Theory Comput., 2008, 4, 819-834.

84 M. R. Machado, E. E. B. Guisasola, F. Klein, M. Sóñora, S. Silva and S. Pantano, J. Chem. Theory Comput., 2019, 15, 2719-2733.

85 E. E. Barrera, M. R. Machado and S. Pantano, J. Chem. Theory Comput., 2019, 15, 5674-5688.
86 H. Berendsen, D. van der Spoel and R. van Drunen, Comput. Phys. Commun., 1995, 91, 43-56.

87 M. J. Abraham, T. Murtola, R. Schulz, S. Páll, J. C. Smith, B. Hess and E. Lindahl, SoftwareX, 2015, 1-2, 1925.

88 M. J. Abraham, D. van der Spoel, E. Lindahl, B. Hess and the GROMACS development team, GROMACS User Manual version 2016, 2018.

89 Schroedinger, LLC, The PyMOL Molecular Graphics System, Version 1.2r3pre.

90 Chemical Computing Group ULC, Molecular Operating Environment (MOE), 2013.08, 2018, 1010 Sherbooke St. West, Suite 910, Montreal, QC, Canada, H3A 2R7.

91 T.-Y. Lin and D. B. Weibel, Appl. Microbiol. Biotechnol., 2016, 100, 4255-4267.

92 A. A. Spector and M. A. Yorek, J. Lipid Res., 1985, 26, 10151035.

93 G. van Meer, D. R. Voelker and G. W. Feigenson, Nat. Rev. Mol. Cell Biol., 2008, 9, 112-124.

94 L. Martínez, R. Andrade, E. G. Birgin and J. M. Martínez, J. Comput. Chem., 2009, 30, 2157-2164.

95 H. Berendsen, J. Postma, W. Van Gunsteren and J. Hermans, Intermolecular Forces, D. Reidel Publishing Company, Dordrecht, 1981, pp. 331-342.

96 G. Bussi, D. Donadio and M. Parrinello, J. Chem. Phys., 2007, 126, 014101.

97 H. J. C. Berendsen, J. P. M. Postma, W. F. van Gunsteren, A. DiNola and J. R. Haak, J. Chem. Phys., 1984, 81, 36843690.

98 M. Parrinello and A. Rahman, J. Appl. Phys., 1981, 52, 7182-7190.

99 U. Essmann, L. Perera, M. L. Berkowitz, T. Darden, H. Lee and L. G. Pedersen, J. Chem. Phys., 1995, 103, 8577-8593.

100 D. H. de Jong, G. Singh, W. F. D. Bennett, C. Arnarez, T. A. Wassenaar, L. V. Schäfer, X. Periole, D. P. Tieleman and S.-J. Marrink, J. Chem. Theory Comput., 2013, 9, 687697.

101 J. A. Graham, J. W. Essex and S. Khalid, J. Chem. Inf. Model., 2017, 57, 650-656.

102 T. A. Wassenaar, H. I. Ingólfsson, R. A. Böckmann, D. P. Tieleman and S. J. Marrink, J. Chem. Theory Comput., 2015, 11, 2144-2155.

103 S. O. Yesylevskyy, L. V. Schäfer, D. Sengupta and S.-J. Marrink, PLoS Comput. Biol., 2010, 6, e1000810.

104 S. Jo, T. Kim and W. Im, PLoS One, 2007, 2, 1-9.

105 I. G. Tironi, R. Sperb, P. E. Smith and W. F. van Gunsteren, J. Chem. Phys., 1995, 102, 5451-5459.

106 E. F. Pettersen, T. D. Goddard, C. C. Huang, G. S. Couch, D. M. Greenblatt, E. C. Meng and T. E. Ferrin, J. Comput. Chem., 2004, 25, 1605-1612.

107 A. Leaver-Fay, M. Tyka, S. M. Lewis, O. F. Lange, J. Thompson, R. Jacak, K. Kaufman, P. D. Renfrew, C. A. Smith, W. Sheffler, I. W. Davis, S. Cooper, A. Treuille, D. J. Mandell, F. Richter, Y.-E. A. Ban, S. J. Fleishman, J. E. Corn, D. E. Kim, S. Lyskov, M. Berrondo, S. Mentzer, Z. Popović, J. J. Havranek, J. Karanicolas, R. Das, J. Meiler, 
T. Kortemme, J. J. Gray, B. Kuhlman, D. Baker and P. Bradley, Methods Enzymol., 2011, 487, 545-574.

108 W. Humphrey, A. Dalke and K. Schulten, J. Mol. Graphics, 1996, 14, 33-38.

109 A. Goldenzweig, M. Goldsmith, S. E. Hill, O. Gertman, P. Laurino, Y. Ashani, O. Dym, T. Unger, S. Albeck, J. Prilusky, R. L. Lieberman, A. Aharoni, I. Silman, J. L. Sussman, D. S. Tawfik and S. J. Fleishman, Mol. Cell, 2016, 63, 337-346.

110 A. Einstein and R. Fürt, Investigations on the theory of Brownian movement, Dover Publications, 1956, p. 119.

111 J. S. Hub, B. L. de Groot and D. van der Spoel, J. Chem. Theory Comput., 2010, 6, 3713-3720.
112 B. Wang, J. Zhang, Y. Zhang, Z. Mao, N. Lu and Q. H. Liu, RSC Adv., 2018, 8, 41517-41525.

113 M. Tang, A. J. Waring and M. Hong, J. Am. Chem. Soc., 2007, 129, 11438-11446.

114 M.-L. Jobin, L. Vamparys, R. Deniau, A. Grélard, C. Mackereth, P. Fuchs and I. Alves, Int. J. Mol. Sci., 2019, 20, 4441.

115 H. Herce, A. Garcia, J. Litt, R. Kane, P. Martin, N. Enrique, A. Rebolledo and V. Milesi, Biophys. J., 2009, 97, 19171925.

116 A. Melcrová, S. Pokorna, S. Pullanchery, M. Kohagen, P. Jurkiewicz, M. Hof, P. Jungwirth, P. S. Cremer and L. Cwiklik, Sci. Rep., 2016, 6, 1-12. 